\title{
INVESTIGATION OF NITROGEN ATOMIZED PM ALLOY 625
}

Frank J. Rizzo and John J. Conway

Crucible Compaction Metals

1001 Robb Hill Road

Oakdale, Pennsylvania 15071

and

Dr. John F. Radavich and Jennifer A. Jones

Purdue University

West Lafayette, Indiana 47906

\begin{abstract}
Unlike argon atomized PM A625(1), the nitrogen atomized powder version of the alloy, PM N625, does not age harden with conventional heat treatment cycles. It is more like conventional cast and wrought alloy 625 in this respect, however, it has some features which are unique to the powder. PM N625 maintains constant mechanical properties after thermal exposure up to $649^{\circ} \mathrm{C}\left(1200^{\circ} \mathrm{F}\right)$ for as long as 1000 hours. This paper discusses the relationships among chemistry, thermal treatment, microstructure, and mechanical properties.
\end{abstract}


Introduction

Several years ago, Crucible began to investigate the possibility of producing mill forms and PM near net shapes from inert gas atomized alloy 625 (i.e., UNS N06625) powder. Unlike its cast and wrought counterpart, it was shown previously ${ }^{(2)}$ that parts produced from argon atomized PM A625 powder, could be precipitation hardened easily with aging times as short as four hours. This aging response in the PM A625 material is the result of a fine precipitation of $\gamma^{\prime \prime}\left(\mathrm{Ni}_{3} \mathrm{Nb}\right)$ and some other phases unique to PM A625 and is aided by the chemical homogeneity of the rapidly solidified powder product. Details of this phenomena are reported elsewhere ${ }^{(3)}$. However, using similar short heat treatment cycles with nitrogen atomized PM N625 does not result in precipitation hardening.

Early work at Crucible was aimed at producing a higher strength alloy 625 material for corrosion resistant applications for the Oil and Gas Industry and therefore, was concentrated on the argon atomized alloy due to the age hardening limitation with nitrogen atomized material. In addition, the microstructure of hot isostatically pressed (i.e., HIP) PM N625 made to the conventional chemistry, exhibited a continuous precipitation of carbides and nitrides at prior particle surfaces. This resulted in lower than desired ductilities and thus, required some chemistry optimization work to be a useful material.

More recently, applications using conventional alloy 625 have been identified where good corrosion resistance at elevated temperature (up to $538^{\circ} \mathrm{C}\left(1000^{\circ} \mathrm{F}\right)$ ) is required. A concern was raised with conventional alloy 625 and its use for extended periods of time at these elevated temperatures because age hardening and subsequent degradation of ductility and corrosion resistance is possible with this material. In addition, good producibility and fabricability (i.e. welding, etc.) is desired. PM N625 is an outstanding candidate for these applications because it is a powder material and can take advantage of all the benefits of powder such as near net shape parts, fine microstructure, good reproducible strength, chemical homogeneity, etc. and is readily weldable.

\section{Initial Work}

In the early 1980's, during the initial Crucible investigations ${ }^{(4)}$ of nitrogen atomized PM N625, the composition was set at the mid-range for all of the elements based on UNS N06625. After the first laboratory heats were made, the microstructural problems with prior particle outlining were discovered and found to be titanium carbides and nitrides. Along with the prior particle outlining, a corresponding decrease in ductility was observed. A heat was made with essentially no titanium and the microstructural and ductility problems were alleviated. The strength and corrosion resistance of the alloy were unchanged ${ }^{(4)}$. From that time on, Crucible has made PM N625 with no titanium addition. A decision was made at that time to make PM N625 without iron as well because it did not enhance either the strength or the corrosion resistance of this alloy. The last of the early compositional findings for PM N625 was to keep the nitrogen content as low as possible. Since the nitrogen is dependent upon the atomization event and the compositional solubility, this element is more difficult to precisely control. Hence the baseline composition was established for PM N625 and is shown as the baseline chemistry in Table I.

TABLE I. - CHEMICAL COMPOSITION OF PM N625 AND CONVENTIONAL ALLOY 625 BAR USED FOR THE THERMAL STABILITY STUDY

\begin{tabular}{||c|c|c|c|c|c|c|c|c|c|c|c|c|c|c||}
\hline \multirow{2}{*}{ Material ID } & \multicolumn{10}{|c|}{ Weight Percent for: } \\
\cline { 2 - 16 } & C & Mn & S & P & Si & Cr & NI & Fe & Mo & Al & Nb & TI & N & 0 \\
\hline $\begin{array}{c}\text { Standard } \\
\text { Wrought Bar }\end{array}$ & 0.02 & 0.11 & $<0.001$ & 0.006 & 0.23 & 21.68 & BAL & 3.58 & 8.77 & 0.30 & 3.52 & 0.21 & 0.013 & 0.0026 \\
\hline $\begin{array}{c}\text { PM N625 } \\
\text { Blend } \\
881 N 415\end{array}$ & 0.012 & $<0.02$ & $<0.001$ & 0.009 & 0.01 & 20.82 & BAL & 0.05 & 8.55 & 0.36 & 3.70 & $<0.01$ & 0.060 & 0.0011 \\
\hline $\begin{array}{c}\text { Baseline } \\
\text { PM N625 }\end{array}$ & 0.02 & LAP & LAP & LAP & LAP & 21.00 & BAL & LAP & 8.80 & 0.35 & 3.60 & LAP & LAP & LAP \\
\hline
\end{tabular}


An extensive investigation was conducted to evaluate the mechanical property stability after long time thermal exposure of the baseline PM N625 composition. Conventional cast and wrought 625 bar material was included in the program for a comparison. Table I contains the chemical analyses of the PM N625 baseline blend (i.e., 89IN415) and the bar material used for this study. A number of $3.6 \mathrm{~kg}$ $(8 \mathrm{lb})$ compacts were HIP to provide material for the evaluation. The variables studied included HIP temperature, powder particle size, annealing treatment versus as-HIP, and thermal exposure time and temperature. The evaluation criteria for thermal stability were room temperature tensile properties and microstructural analyses before and after thermal exposure. Good thermal stability would be exhibited by material that did not significantly change in strength or ductility after exposure to elevated temperatures for extended periods of time. The room temperature tensile results from this investigation are presented in Tables II through IV. The as-HIP data appear in Table II, the HIP plus annealed data are in Table III, and the conventional cast and wrought bar data are in Table IV.

HIP temperatures of $1130^{\circ} \mathrm{C}\left(2065^{\circ} \mathrm{F}\right)$ and $1163^{\circ} \mathrm{C}\left(2125^{\circ} \mathrm{F}\right)$ were evaluated in this study. A comparison of the data in Table II shows that in this range of HIP temperature, there was no effect on the thermal stability of PM N625 at $315^{\circ} \mathrm{C}\left(600^{\circ} \mathrm{F}\right)$ and $427^{\circ} \mathrm{C}\left(800^{\circ} \mathrm{F}\right)$ for exposure times up to 1000 hours. However, after a $649^{\circ} \mathrm{C}\left(1200^{\circ} \mathrm{F}\right) / 1000 \mathrm{hrs}$ treatment, the ductility of the material HIP at $1163^{\circ} \mathrm{C}\left(2125^{\circ} \mathrm{F}\right)$ decreased more than that of the material HIP at $1130^{\circ} \mathrm{C}\left(2065^{\circ} \mathrm{F}\right)$. This effect is believed to be due to additional precipitation caused by increased solutioning during the higher temperature HIP process. The effect of the $1149^{\circ} \mathrm{C}\left(2100^{\circ} \mathrm{F}\right)$ solution annealing treatment versus the as-HIP material was the same as the higher HIP temperature material, probably for the same reason. These effects could not be confirmed by light microscopy due to the small size of the precipitates.

TABLE II. - ROOM TEMPERATURE TENSILE PROPERTIES OF AS-HIP PM N625 IN VARIOUS THERMALLY TREATED CONDITIONS

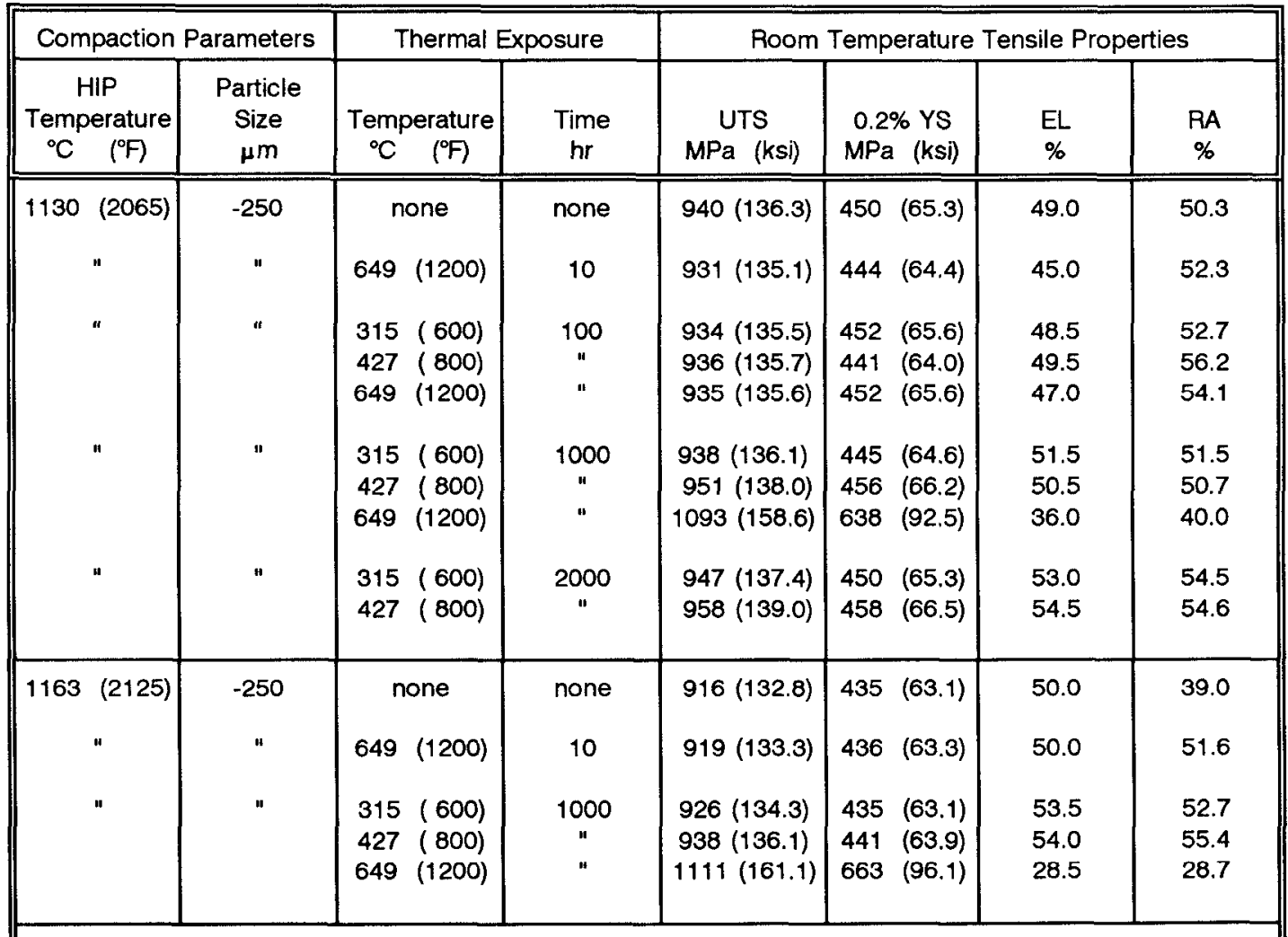

Note: All results represent the average of two tests. 
Powder particle sizes ranging from $-150 \mu \mathrm{m}$ to $-1000 \mu \mathrm{m}$ were investigated and the results are given in Table III. All of this PM N625 material was evaluated in the HIP plus annealed condition. No significant effect of powder particle size was observed on thermal exposure at $315^{\circ} \mathrm{C}\left(600^{\circ} \mathrm{F}\right)$ and $427^{\circ} \mathrm{C}\left(800^{\circ} \mathrm{F}\right)$ for times up to 2000 hours. However, after exposure at $649^{\circ} \mathrm{C}\left(1200^{\circ} \mathrm{F}\right) / 1000 \mathrm{hrs}$, the strength increased significantly and the ductility decreased to about half the as-HIP levels for all particle sizes studied. No significant differences were found in either strength or ductility that were related only to particle size variations. Likewize, annealing at $1149^{\circ} \mathrm{C}\left(2100^{\circ} \mathrm{F}\right)$ showed no significant differences of strength or ductility after thermal exposure from as-HIP PM N625 material.

TABLE III. - ROOM TEMPERATURE TENSILE PROPERTIES OF ANNEALED PM N625 IN VARIOUS THERMALLY TREATED CONDITIONS

\begin{tabular}{|c|c|c|c|c|c|c|c|}
\hline \multirow{2}{*}{$\begin{array}{c}\text { HIP } \\
\text { Temperatures } \\
C^{\circ}\left(F^{\circ}\right)\end{array}$} & \multirow{2}{*}{$\begin{array}{l}\text { Particle } \\
\text { Size } \\
\mu \mathrm{m}\end{array}$} & \multicolumn{2}{|c|}{ Thermal Exposure } & \multicolumn{4}{|c|}{ Room Temperature Tensile Properties } \\
\hline & & $\begin{array}{c}\text { Temperature } \\
\mathrm{C}^{\circ} \quad\left(\mathrm{F}^{\circ}\right)\end{array}$ & $\begin{array}{c}\text { Time } \\
\mathrm{hr}\end{array}$ & $\begin{array}{c}\text { UTS } \\
\mathrm{MPa}(\mathrm{ksi})\end{array}$ & $\begin{array}{l}0.2 \% \text { YS } \\
\mathrm{MPa} \text { (ksi) }\end{array}$ & $\begin{array}{l}E L \\
\%\end{array}$ & $\begin{array}{l}\text { RA } \\
\%\end{array}$ \\
\hline $1130(2065)$ & -1000 & none & none & $898(130.3)$ & $415(60.2)$ & 47.0 & 57.4 \\
\hline$"$ & $"$ & $649(1200)$ & 1000 & $1104(160.2)$ & $669(97.1)$ & 29.5 & 29.1 \\
\hline$"$ & $"$ & $\begin{array}{l}315(600) \\
427(800)\end{array}$ & 2000 & $\begin{array}{l}911(132.2) \\
940(136.3)\end{array}$ & $\begin{array}{l}423(61.4) \\
441(64.0)\end{array}$ & $\begin{array}{l}52.0 \\
51.0\end{array}$ & $\begin{array}{l}59.6 \\
53.9\end{array}$ \\
\hline$"$ & -500 & none & none & $901(130.7)$ & $414(60.1)$ & 49.5 & 61.2 \\
\hline " & $"$ & $649(1200)$ & 1000 & $1109(160.9)$ & $684(99.2)$ & 27.0 & 32.2 \\
\hline$"$ & $"$ & $\begin{array}{l}315(600) \\
427(800)\end{array}$ & 2000 & $\begin{array}{l}924(134.0) \\
948(137.5)\end{array}$ & $\begin{array}{l}431(62.5) \\
449(65.1)\end{array}$ & $\begin{array}{l}51.5 \\
51.5\end{array}$ & $\begin{array}{l}57.6 \\
60.0\end{array}$ \\
\hline$"$ & -250 & none & none & $929(134.7)$ & $443(64.2)$ & 53.0 & 54.0 \\
\hline$"$ & $"$ & $649(1200)$ & 10 & $915(132.7)$ & $427(61.9)$ & 50.0 & 54.7 \\
\hline$n$ & $"$ & $315(600)$ & 100 & $906(131.4)$ & $414(60.1)$ & 52.5 & 58.7 \\
\hline$n$ & $n$ & $\begin{array}{l}315(600) \\
427(800) \\
649(1200)\end{array}$ & $\begin{array}{c}1000 \\
" \\
"\end{array}$ & $\begin{array}{c}907(131.6) \\
934(135.5) \\
1133(164.3)\end{array}$ & $\begin{array}{c}420(60.9) \\
435(63.1) \\
698(101.3)\end{array}$ & $\begin{array}{l}56.0 \\
54.5 \\
34.5\end{array}$ & $\begin{array}{l}59.0 \\
53.2 \\
34.3\end{array}$ \\
\hline$"$ & $"$ & $315(600)$ & 2000 & $918(133.2)$ & $416(60.4)$ & 56.0 & 57.9 \\
\hline$"$ & -150 & none & none & $907(131.6)$ & $425(61.6)$ & 51.0 & 59.7 \\
\hline$"$ & $"$ & $649(1200)$ & 1000 & $1113(161.5)$ & $683(99.0)$ & 31.0 & 35.3 \\
\hline " & " & $\begin{array}{l}315(600) \\
427(800)\end{array}$ & 2000 & $\begin{array}{l}932(135.2) \\
931(135.1)\end{array}$ & $\begin{array}{l}436(63.2) \\
436(63.3)\end{array}$ & $\begin{array}{l}49.5 \\
50.5\end{array}$ & $\begin{array}{l}57.1 \\
57.2\end{array}$ \\
\hline $\begin{array}{l}\text { 1. The } \\
\text { 2. All } r\end{array}$ & $\begin{array}{l}\text { tre } \\
\text { res }\end{array}$ & age $\mathrm{me}$ & 11 & PF/1 h & & & \\
\hline
\end{tabular}

An analysis of the data in Tables II and III shows the dramatic effects of thermal exposure time and temperature on PM N625. Up to $427^{\circ} \mathrm{C}\left(800^{\circ} \mathrm{F}\right)$, for exposures as long as 2000 hours, there was no significant change in strength or ductility. But, at $649^{\circ} \mathrm{C}\left(1200^{\circ} \mathrm{F}\right)$, after 1000 hours the material aged without regard to any other process variation. This effect was not evident after 100 hours of exposure at $649^{\circ} \mathrm{C}\left(1200^{\circ} \mathrm{F}\right)$. Figure 1 shows these trends for $0.2 \%$ yield strength and reduction of area values for baseline PM N625 HIP at $1130^{\circ} \mathrm{C}\left(2065^{\circ} \mathrm{F}\right)$ with a powder particle size of $-250 \mu \mathrm{m}$ which was not annealed prior to thermal exposure. 


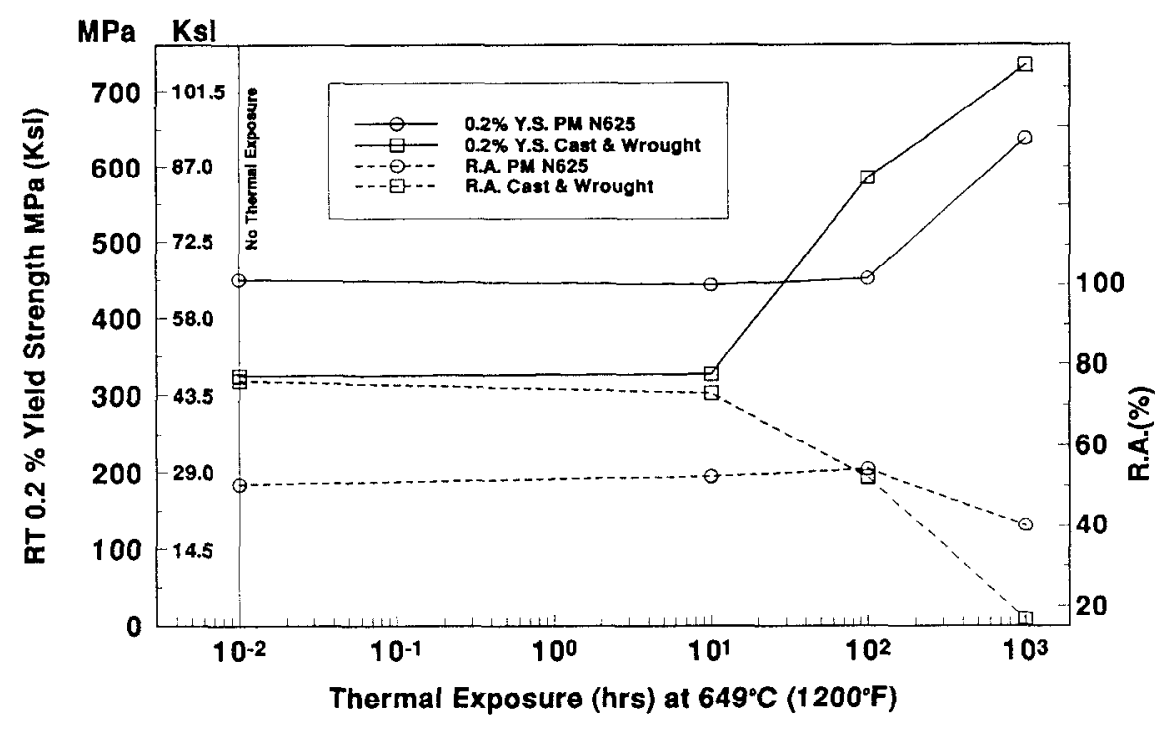

Figure 1. Thermal Exposure at $649^{\circ} \mathrm{C}\left(1200^{\circ} \mathrm{F}\right)$ versus $0.2 \%$ Yield Strength at Room Temperature for Baseline PM N625 and Conventional 625 Bar Material.

The conventional cast and wrought 625 bar material, received in the solution annealed condition, also exhibited no significant mechanical property differences at $315^{\circ} \mathrm{C}\left(600^{\circ} \mathrm{F}\right)$ and $427^{\circ} \mathrm{C}\left(800^{\circ} \mathrm{F}\right)$ for times as long as 2000 hours. These results are shown in Table IV. However, at $649^{\circ} \mathrm{C}\left(1200^{\circ} \mathrm{F}\right)$, age hardening began to take place in less than 100 hours and after 1000 hours, the strength increased almost 2-1/2 times its as-received value with a corresponding decrease in ductility to about $1 / 4$ of its as-received level. These strength and ductility data are also plotted in Figure 1 along with the PM N625 results.

TABLE IV. - ROOM TEMPERATURE TENSILE PROPERTIES OF ANNEALED CONVENTIONAL ALLOY 625 BAR IN VARIOUS THERMALLY TREATED CONDITIONS

\begin{tabular}{|c|c|c|c|c|c|}
\hline \multicolumn{2}{|c|}{ Thermal Exposure } & \multicolumn{4}{|c|}{ Room Temperature Tensile Properties } \\
\hline $\begin{array}{c}\text { Temperature } \\
\mathrm{C}^{\circ}\left(\mathrm{F}^{\circ}\right)\end{array}$ & $\begin{array}{c}\text { Time } \\
\mathrm{hr}\end{array}$ & $\begin{array}{c}\text { UTS } \\
\text { MPa (ksi) }\end{array}$ & $\begin{array}{c}0.2 \% \text { YS } \\
\mathrm{MPa} \text { (ksi) }\end{array}$ & $\begin{array}{l}E L \\
\%\end{array}$ & $\begin{array}{l}\text { RA } \\
\%\end{array}$ \\
\hline none & none & $738(107.0)$ & $324(47.0)$ & 68.5 & 75.8 \\
\hline $649(1200)$ & 10 & $741(107.3)$ & $327(47.4)$ & 72.0 & 72.9 \\
\hline $\begin{array}{l}315(600) \\
427(800) \\
649(1200)\end{array}$ & $\begin{array}{c}100 \\
" \\
"\end{array}$ & $\begin{array}{l}741(107.5) \\
741(107.3) \\
927(134.4)\end{array}$ & $\begin{array}{l}322(46.7) \\
325(47.1) \\
585(84.9)\end{array}$ & $\begin{array}{l}71.5 \\
72.0 \\
51.0\end{array}$ & $\begin{array}{l}74.7 \\
74.5 \\
52.1\end{array}$ \\
\hline $\begin{array}{l}315(600) \\
427(800) \\
649(1200)\end{array}$ & $\begin{array}{c}1000 \\
" \\
"\end{array}$ & $\begin{array}{l}733(106.3) \\
737(106.9) \\
953(138.3)\end{array}$ & $\begin{array}{c}316(45.8) \\
322(46.7) \\
733(106.3)\end{array}$ & $\begin{array}{l}77.5 \\
77.5 \\
13.0\end{array}$ & $\begin{array}{l}76.2 \\
74.7 \\
16.7\end{array}$ \\
\hline $\begin{array}{l}315(600) \\
427(800)\end{array}$ & $\begin{array}{c}2000 \\
" ~\end{array}$ & $\begin{array}{l}734(106.5) \\
738(107.1)\end{array}$ & $\begin{array}{l}316(45.9) \\
315(45.7)\end{array}$ & $\begin{array}{l}73.5 \\
76.0\end{array}$ & $\begin{array}{l}75.6 \\
74.6\end{array}$ \\
\hline
\end{tabular}


All of the material included in this investigation was thoroughly evaluated metallographically. Slightly coarser microstructure was noted with higher HIP temperature, solution annealing treatment, and larger powder particle size. This did not manifest itself in perceptible differences in mechanical properties. No other microstructural differences could be detected by light microscopy. Typical microstructures of blend 89IN415, the PM N625 baseline chemistry, after thermal exposures are shown in Figure 2.

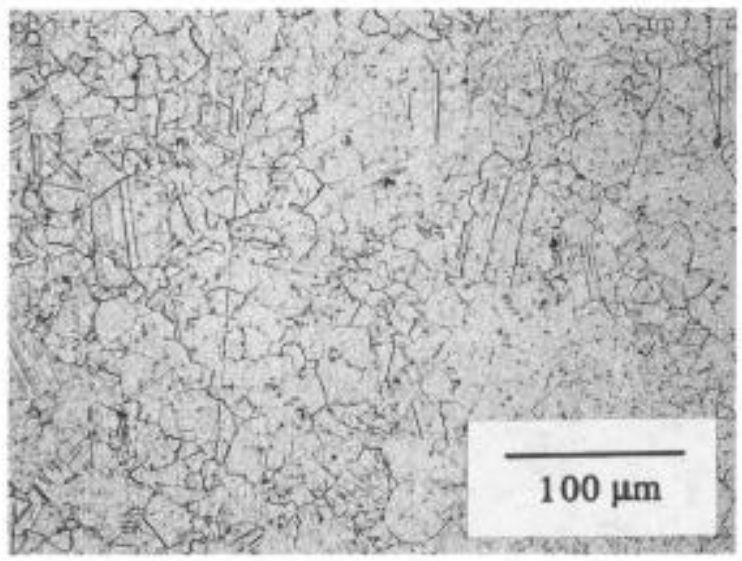

$315^{\circ} \mathrm{C}\left(600^{\circ} \mathrm{F}\right) / 100 \mathrm{hrs}$

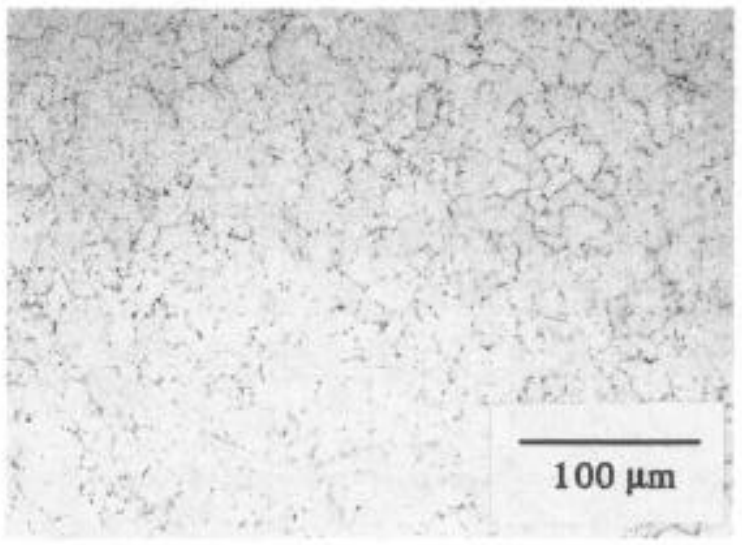

$649^{\circ} \mathrm{C}\left(1200^{\circ} \mathrm{F}\right) / 1000 \mathrm{hrs}$

Figure 2. HIP Plus Thermally Exposed Microstructures of PM N625. Etchant: Kallings

The conventional cast and wrought alloy 625 bar did not show any evidence of discrete precipitation and contained extremely large grains by comparison with the PM N625 material. The grain boundaries of this material get wider with longer thermal exposure times and contain phase precipitation not present in the as-received bar. Typical microstructures of the conventional bar after exposure are pictured in Figure 3. 


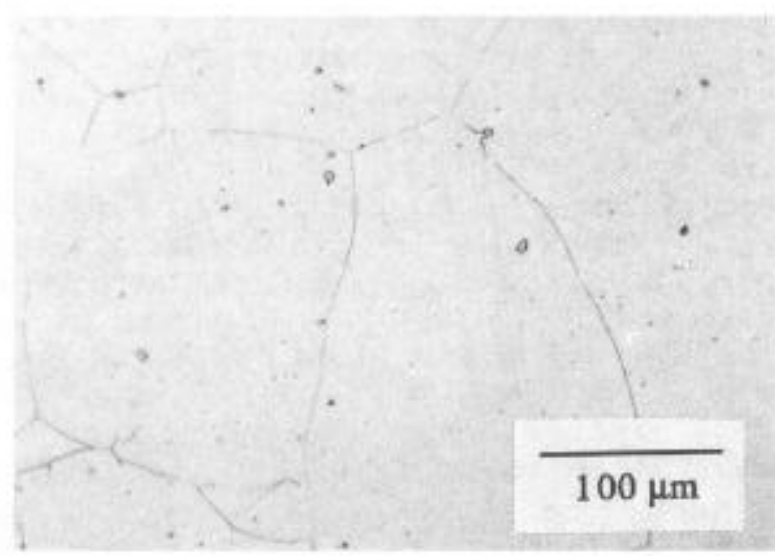

$649^{\circ} \mathrm{C}\left(1200^{\circ} \mathrm{F}\right) / 10 \mathrm{hrs}$

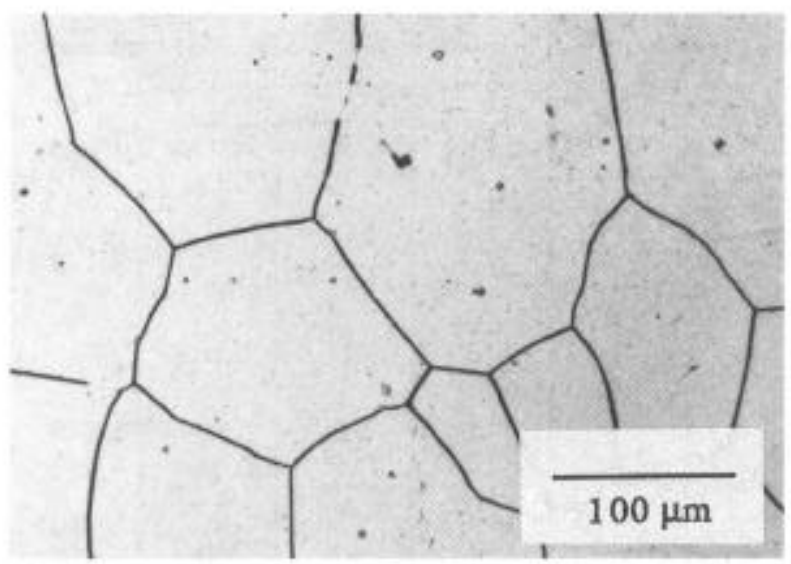

$649^{\circ} \mathrm{C}\left(1200^{\circ} \mathrm{F}\right) / 100 \mathrm{hrs}$

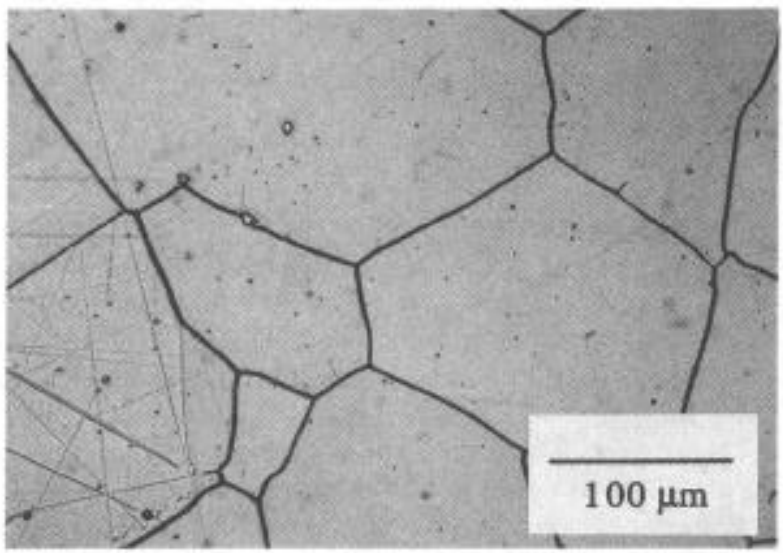

$649^{\circ} \mathrm{C}\left(1200^{\prime} \mathrm{F}\right) / 1000 \mathrm{hrs}$

Figure 3. Typical Microstructure of Conventional Alloy 625 Bar after Thermal Exposure at $649^{\circ} \mathrm{C}\left(1200^{\circ} \mathrm{F}\right)$. Etchant: Kallings 


\section{Chemistry Optimization Study}

As indicated in the early studies, minor chemistry modifications were required to optimize the thermal stability of the PM N625 material and a program was initiated to accomplish this goal. The evaluation criterion for this work was a thermal exposure at $649^{\circ} \mathrm{C}\left(1200^{\circ} \mathrm{F}\right)$ for 1000 hours because this was the only condition from the earlier program to consistently show a hardening response. The titanium and iron contents of the baseline PM N625 composition were set to be as low as possible (i.e., no intentional additions). The particle size for this work was set at $-250 \mu \mathrm{m}$ and two HIP temperatures were used; $1130^{\circ} \mathrm{C}\left(2065{ }^{\circ} \mathrm{F}\right)$ and $1182^{\circ} \mathrm{C}\left(2160^{\circ} \mathrm{F}\right)$. Solution annealing of the PM N625 did not show a benefit to properties before or after thermal exposure so none was included in this study.

Eight $22.7 \mathrm{~kg}(50 \mathrm{lb})$ heats were melted and atomized to look at the effects of carbon, nitrogen, iron, niobium, and aluminum. Table $V$ lists the actual chemical analyses for these heats. The powder was screened to $-250 \mu \mathrm{m}$ particle size and processed through consolidation with some material being HIP at $1130^{\circ} \mathrm{C}\left(2065^{\circ} \mathrm{F}\right)$ and some bring HIP at $1182^{\circ} \mathrm{C}\left(2160^{\circ} \mathrm{F}\right)$. All compacts were analyzed for density and thermally induced porosity to verity that the processing was done correctly.

TABLE V. - CHEMISTRY OF PM N625 MODIFICATIONS FOR THE COMPOSITION OPTIMIZATION STUDY

\begin{tabular}{|c|c|c|c|c|c|c|c|c|c|}
\hline \multirow{2}{*}{$\begin{array}{l}\text { Weight } \\
\text { Percent } \\
\text { for: }\end{array}$} & \multirow[b]{2}{*}{ Nominal } & \multicolumn{8}{|c|}{ Heat Number } \\
\hline & & L21 & L26 & L3 & L10 & L22 & L23 & L24 & L25 \\
\hline c & 0.020 & 0.016 & 0.011 & 0.007 & 0.003 & 0.057 & 0.023 & 0.016 & 0.019 \\
\hline $\mathbf{N}$ & LAP & 0.0696 & 0.1750 & 0.0490 & 0.0632 & 0.0756 & 0.0818 & 0.0681 & 0.0806 \\
\hline $\mathrm{Fe}$ & LAP & 0.04 & 1.91 & 0.05 & 0.09 & 0.05 & 0.04 & 2.76 & 0.08 \\
\hline $\mathrm{Nb}$ & 3.60 & 3.57 & 3.66 & 3.12 & 3.74 & 3.56 & 3.53 & 3.54 & 4.08 \\
\hline Al & 0.35 & $<0.01$ & $<0.01$ & $<0.01$ & $<0.01$ & $<0.01$ & 0.09 & $<0.01$ & $<0.01$ \\
\hline $\mathrm{Ti}$ & LAP & $<0.01$ & $<0.01$ & $<0.01$ & $<0.01$ & $<0.01$ & $<0.01$ & $<0.01$ & $<0.01$ \\
\hline$s$ & LAP & 0.001 & 0.002 & 0.002 & 0.002 & 0.001 & 0.001 & 0.001 & 0.001 \\
\hline Mn & LAP & $<0.01$ & $<0.01$ & $<0.01$ & $<0.01$ & $<0.01$ & $<0.01$ & $<0.01$ & $<0.01$ \\
\hline $\mathrm{Si}$ & LAP & $<0.01$ & 0.14 & 0.01 & 0.01 & 0.01 & 0.01 & 0.01 & 0.01 \\
\hline P & LAP & 0.01 & 0.01 & 0.01 & 0.01 & 0.01 & 0.01 & 0.01 & 0.01 \\
\hline $\mathrm{Cr}$ & 21.00 & 21.11 & 20.88 & 21.37 & 20.73 & 20.82 & 20.95 & 20.90 & 20.70 \\
\hline Mo & 8.80 & 8.60 & 8.71 & 8.52 & 8.51 & 8.68 & 8.61 & 8.70 & 8.59 \\
\hline Co & LAP & $<0.01$ & $<0.01$ & $<0.01$ & $<0.01$ & $<0.01$ & $<0.01$ & $<0.01$ & $<0.01$ \\
\hline 0 & LAP & 0.0100 & 0.0167 & 0.0189 & 0.0148 & 0.0086 & 0.0084 & 0.0107 & 0.0096 \\
\hline $\mathrm{Ni}$ & BAL & BAL & BAL & BAL & BAL & BAL & BAL & BAL & BAL \\
\hline
\end{tabular}

Duplicate room temperature tensile tests were run for each heat and HIP temperature variation in the as-HIP and thermally exposed $\left.\left(649^{\circ} \mathrm{C}\left(1200^{\circ} \mathrm{F}\right) / 1000 \mathrm{hrs}\right)\right)$ conditions. The data for all heats in the asHIP condition appear in Table VI. These results show little difference among the eight heats. Higher nitrogen (L26) and higher niobium (L25) contents exhibit slight increases in strength probably due to solid solution strengthening and/or nitride precipitation. Strength levels for the higher HIP temperature compacts for all chemistry modifications were significantly lower than their lower temperature HIP counterparts. Higher HIP temperature improved the ductility values for some of the modifications and decreased them for others. The best combination of strength and ductility was exhibited by heats $L 3$ and L10 where all variable elements were at the low end of the acceptable range with the exception of the niobium content of heat L10. 
TABLE VI. - ROOM TEMPERATURE TENSILE PROPERTY OF AS-HIP PM N625 COMPOSITION VARIATIONS

\begin{tabular}{|c|c|c|c|c|c|}
\hline $\begin{array}{l}\text { Heat } \\
\text { Number }\end{array}$ & $\begin{array}{l}\text { HIP Temperature } \\
{ }^{\circ} \mathrm{C} \quad\left({ }^{\circ} \mathrm{F}\right)\end{array}$ & \begin{tabular}{cc}
\multicolumn{2}{c}{ UTS } \\
$\mathrm{MPa}$ & (ksi)
\end{tabular} & $\begin{array}{c}0.2 \% \text { YS } \\
\mathrm{MPa} \quad \text { (ksi) }\end{array}$ & $\begin{array}{l}E L \\
\%\end{array}$ & $\begin{array}{l}\text { RA } \\
\%\end{array}$ \\
\hline $\begin{array}{l}\text { L10 } \\
\text { L21 } \\
\text { L22 } \\
\text { L23 } \\
\text { L24 } \\
\text { L25 } \\
\text { L26 } \\
\text { L3 }\end{array}$ & $\begin{array}{c}1130 \text { (2065) } \\
" \\
" \\
" \\
" \\
" \\
"\end{array}$ & $\begin{array}{ll}957 & (138.8) \\
965 & (139.9) \\
968 & (140.4) \\
967 & (140.2) \\
946 & (137.2) \\
982 & (142.5) \\
997 & (144.6) \\
938 & (136.1)\end{array}$ & $\begin{array}{ll}462 & (67.0) \\
461 & (66.8) \\
461 & (66.9) \\
462 & (67.0) \\
446 & (64.7) \\
477 & (69.2) \\
506 & (73.4) \\
450 & (65.2)\end{array}$ & $\begin{array}{l}45.5 \\
42.5 \\
40.0 \\
41.0 \\
40.5 \\
42.0 \\
40.5 \\
45.5\end{array}$ & $\begin{array}{l}45.4 \\
42.2 \\
42.9 \\
41.6 \\
41.0 \\
47.5 \\
42.2 \\
54.8\end{array}$ \\
\hline $\begin{array}{l}\text { L10 } \\
\text { L21 } \\
\text { L22 } \\
\text { L23 } \\
\text { L24 } \\
\text { L25 } \\
\text { L26 } \\
\text { L3 }\end{array}$ & $\begin{array}{c}1182 \text { (2160) } \\
" \\
" \\
" \\
" \\
" \\
"\end{array}$ & $\begin{array}{ll}905 & (131.3) \\
910 & (132.0) \\
922 & (133.7) \\
925 & (134.1) \\
902 & (130.8) \\
931 & (135.0) \\
951 & (138.0) \\
889 & (128.9)\end{array}$ & $\begin{array}{ll}394 & (57.2) \\
400 & (58.0) \\
402 & (58.3) \\
401 & (58.2) \\
392 & (56.8) \\
409 & (59.3) \\
411 & (59.6) \\
390 & (56.5)\end{array}$ & $\begin{array}{l}54.5 \\
40.0 \\
45.0 \\
43.5 \\
43.5 \\
38.0 \\
39.5 \\
50.5\end{array}$ & $\begin{array}{l}52.5 \\
37.9 \\
47.0 \\
39.4 \\
44.4 \\
34.5 \\
39.5 \\
56.1\end{array}$ \\
\hline
\end{tabular}

Table VII contains the room temperature tensile results for all of the heats after thermal exposure. While all compositions showed at least a slight increase in strength with exposure, they did not always have a corresponding decrease in ductility. Heat L25 (i.e., $4.08 \% \mathrm{Nb}$ material) exhibited the largest increase in strength (i.e., approximately $276 \mathrm{MPa}(40 \mathrm{ksi}$ ) change in $0.2 \%$ yield strength) and a significant decrease in ductility. Both effects were more pronounced at the lower HIP temperature. Heat L3 (i.e., 3.12\% niobium) showed the smallest increase in strength at the low HIP temperature and it had a slight increase in ductility. The heat (i.e., L10) with the second highest niobium content (i.e., $3.74 \%$ ) had the second highest increase in strength at the lower HIP temperature for all variations studied. This hardening effect was thought ${ }^{(5)}$ to be the result of increased precipitation of $\gamma^{\prime \prime}$ with increasing niobium content. None of the other elemental modifications showed this type of correlation.

TABLE VII. - ROOM TEMPERATURE TENSILE PROPERTIES OF PM N625 COMPOSITION VARIATIONS AFTER $649^{\circ} \mathrm{C}\left(1200^{\circ} \mathrm{F}\right) / 1000 \mathrm{HR}$ EXPOSURE

\begin{tabular}{|c|c|c|c|c|c|}
\hline $\begin{array}{l}\text { Heat } \\
\text { Number }\end{array}$ & $\begin{array}{l}\text { HIP Temperature } \\
{ }^{\circ} \mathrm{C} \quad\left({ }^{\circ} \mathrm{F}\right)\end{array}$ & $\begin{array}{c}\text { UTS } \\
\mathrm{MPa} \quad \text { (ksi) }\end{array}$ & $\begin{array}{cc}0.2 \% & \text { YS } \\
\mathrm{MPa} & (\mathrm{ksi})\end{array}$ & $\begin{array}{l}E L \\
\%\end{array}$ & $\begin{array}{l}\text { RA } \\
\%\end{array}$ \\
\hline $\begin{array}{l}\text { L10 } \\
\text { L21 } \\
\text { L22 } \\
\text { L23 } \\
\text { L24 } \\
\text { L25 } \\
\text { L26 } \\
\text { L3 }\end{array}$ & $\begin{array}{c}1130 \text { (2065) } \\
" \\
" \\
" \\
" \\
" \\
"\end{array}$ & $\begin{array}{rr}1048 & (152.0) \\
980 & (142.2) \\
976 & (141.5) \\
985 & (142.8) \\
960 & (139.3) \\
1164 & (168.9) \\
1002 & (145.4) \\
933 & (135.3)\end{array}$ & $\begin{array}{rr}596 & (86.4) \\
496 & (71.9) \\
487 & (70.6) \\
492 & (71.4) \\
483 & (70.0) \\
764 & (110.8) \\
530 & (76.9) \\
463 & (67.2)\end{array}$ & $\begin{array}{l}38.0 \\
41.5 \\
39.0 \\
38.5 \\
39.5 \\
26.5 \\
35.5 \\
46.0\end{array}$ & $\begin{array}{l}47.4 \\
46.1 \\
38.8 \\
42.8 \\
43.4 \\
31.4 \\
43.1 \\
56.0\end{array}$ \\
\hline $\begin{array}{l}\text { L10 } \\
\text { L21 } \\
\text { L22 } \\
\text { L23 } \\
\text { L24 } \\
\text { L25 } \\
\text { L26 } \\
\text { L3 }\end{array}$ & $\begin{array}{c}1182 \text { (2160) } \\
" \\
" \\
" \\
" \\
" \\
"\end{array}$ & $\begin{aligned} 961 & (139.4) \\
920 & (133.4) \\
921 & (133.6) \\
927 & (134.5) \\
980 & (142.1) \\
1093 & (158.6) \\
945 & (137.1) \\
888 & (128.8)\end{aligned}$ & $\begin{array}{ll}483 & (70.0) \\
421 & (61.1) \\
416 & (60.3) \\
418 & (60.7) \\
523 & (75.9) \\
673 & (97.6) \\
426 & (61.8) \\
407 & (59.0) \\
\end{array}$ & $\begin{array}{l}41.5 \\
45.0 \\
46.0 \\
42.5 \\
33.5 \\
29.5 \\
36.0 \\
47.5\end{array}$ & $\begin{array}{l}49.5 \\
47.4 \\
53.6 \\
44.8 \\
34.4 \\
33.7 \\
38.9 \\
51.9\end{array}$ \\
\hline
\end{tabular}


Light microstructure specimens were examined for all chemistry variations in all conditions of processing and exposure. In general, the higher HIP temperature material exhibited a slightly coarser grain size (e.g., ASTM \#7 versus ASTM \#8). This is probably due to increased solutioning of carbides and/or nitrides in the microstructure. The finest grain size (i.e., ASTM \#9) was noted on heat L26 which contained the highest nitrogen level (i.e., $0.175 \% \mathrm{~N}$ ) in the program. More nitrides resulted in a greater grain boundary pinning effect than in the lower nitrogen heats. The constituents in all of the modification microstructures were similar. Because of the fine size of the precipitates in these materials, light microscopy could not resolve the features which control the relationships between microstructure and mechanical properties.

The thermally exposed material showed the same microstructural characteristics as the as-HIP material. Light microscopy is not able to resolve the precipitation differences which have occurred to account for the observed mechanical property differences. To determine microstructural precipitates in these compositional modifications of PM N625, sample material was taken from heats L3 (i.e., 3.12\% Nb), L10 (i.e., 3.74\% Nb), and L25 (i.e., 4.08\% Nb) for SEM analyses. The specimens were polished through $6 \mu \mathrm{m}$ diamond paste and electropolished in $10 \% \mathrm{HCl}-$ methanol at $25 \mathrm{~V}$ for 10 seconds. Etching was accomplished in the same solution at $5 \mathrm{~V}$ for 2 seconds to put carbides and nitrides in relief. The specimens were additionally etched in a $\mathrm{CrO}_{3}$ solution at $5 \mathrm{~V}$ for 10 seconds to put any $\gamma^{\prime}$ present in relief and remove any $\mathrm{Cr}$-rich particles.

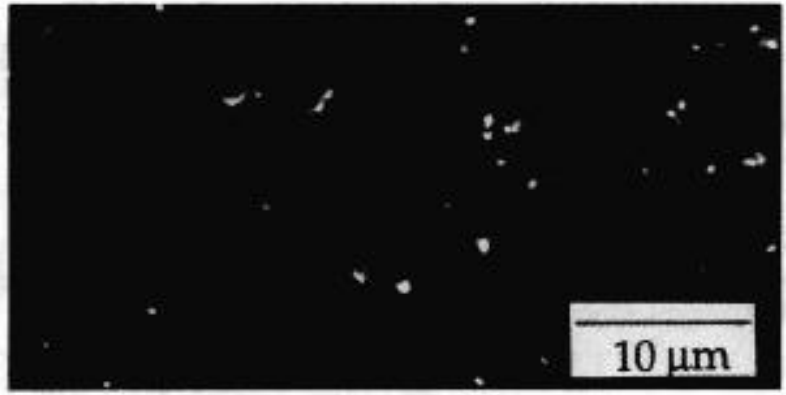

Heat L3

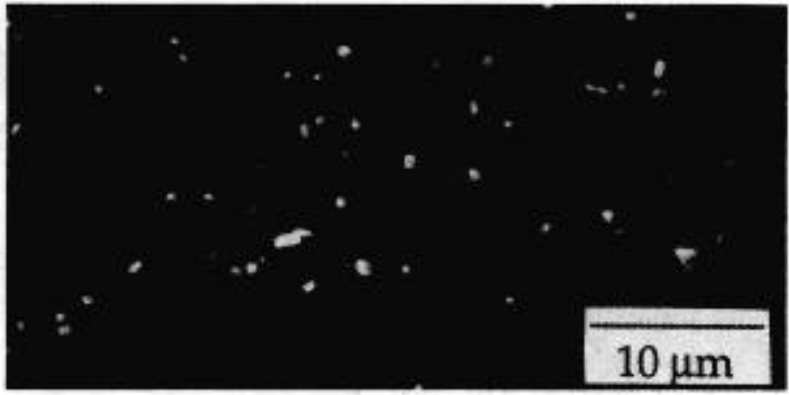

Heat L10

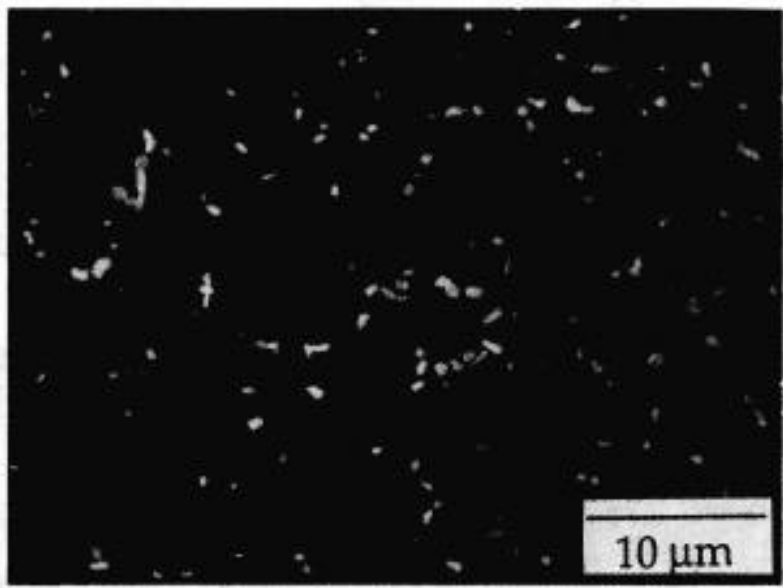

Heat L25

Figure 4. SEM Photographs Showing the Nitride Precipitates in PM N625 Modifications in the As-HIP Condition. Etchant: $10 \% \mathrm{HCl}$ in Methanol Electrolytic 
Figure 4 shows the as-HIP SEM photomicrographs of these three modifications. Fine discrete nitride particles are exhibited. The greater the niobium content the greater the amount of nitride precipitation. Electrolytic extraction and X-ray diffraction analyses of the residues identified the nitride as $\mathrm{CrNbN}$ with a sizable solubility for molybdenum. No carbides or $\gamma$ precipitation was found in the as-HIP material. Work reported ${ }^{(3)}$ on argon atomized PM A625 showed that titanium is needed to precipitate MC carbides which aid in the aging of that material. Since PM N625 lacks titanium, no MC carbide is precipitated and no age hardening can take place with short aging time heat treatments. This same result was observed in argon atomized PM A625 without titanium.

Phase identification work ${ }^{(5)}$ on the thermally exposed specimens could not positively identify $\gamma^{*}$ as the precipitate exhibited in the matrix areas of this material because of its small size. Figure 5 shows the SEM photomicrographs of thermally exposed material from heats L3, L10 and L25. The amount of the fine precipitation increases with increasing niobium content and there is a precipitate depleted region around the $\mathrm{CrNbN}$ nitride particles. This lends credence to the belief that these precipitates are $\gamma$ and that they are responsible for the increase in strength and decrease in ductility after thermal exposure.

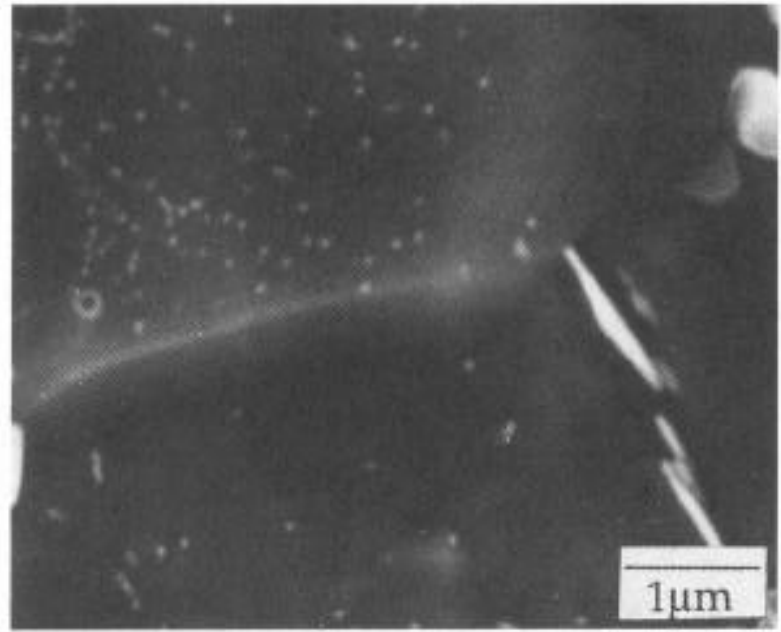

Heat L3

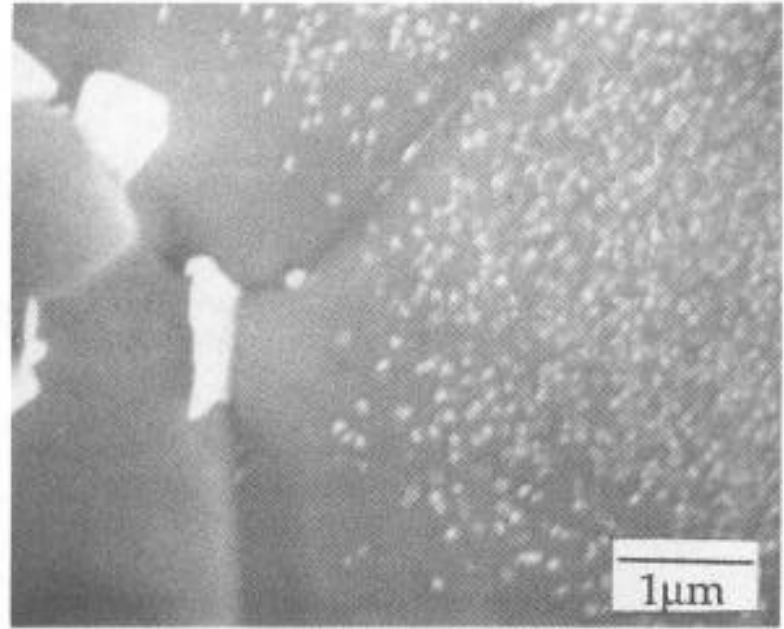

Heat L10

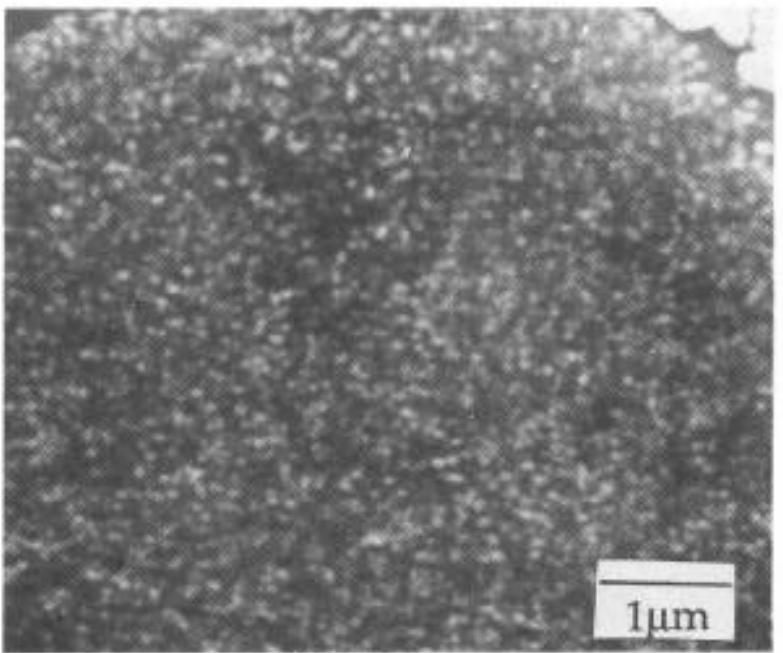

Heat L25

Figure 5. SEM Photographs Showing Fine Precipitates in PM N625 Modifications after Thermal Exposure at $649^{\circ} \mathrm{C}\left(1200^{\circ} \mathrm{F}\right) / 1000 \mathrm{hrs}$. Etchant: $10 \% \mathrm{HCl}$ in Methanol Electrolytic plus $\mathrm{CrO}_{3}$ 


\section{Summary}

This work indicates that the most thermally stable (i.e., least change in mechanical properties after thermal exposure) PM N625 composition is obtained by keeping the level of carbon, nitrogen, iron, aluminum, titanium, and niobium at the low end of the standard specified chemistry range for UNS N06625. It is especially important to minimize the titanium and niobium contents. There may be other reasons to adjust the composition, but these are the guidelines when enhanced thermal stability is desired.

The conventional cast and wrought bar material included in this study was not thermally stable by the criteria used.

\section{Acknowledgements}

The phase identification work included in this report was done by Ms. J. A. Jones as part her Senior Design Project at Purdue University for Dr. J. F. Radavich. Ms. Jones now works for AlliedSignal in Phoenix, Arizona.

\section{References}

1. F. J. Rizzo, "Applicability of rapidly solidified PM Alloy HIP Components", Industrial Heating October, 1986.

2. J. E. Stulga and B. J. McTiernan, "The Metallurgy and Applications of Rapidly Solidified Alloy 625" (Paper Presented at the International Conference of Rapidly Solidified Materials, San Diego, California, 3-5 February 1985), 397.

3. F. J. Rizzo, "Strengthening Precipitates in Argon Atomized PM Alloy A625" (Paper presented at the International Symposium on Superalloys 718, 625, 706 and Derivatives, Pittsburgh, Pennsylvania, 27-29 June 1994).

4. J. E. Stulga and J. J. Eckenrod, "Mechanical Properties and Corrosion Resistance of Powder Metallurgy Inconel 625" (Crucible Research Report 09-275-1, 9/20/82).

5. J. A. Jones, "A Study on the Effect of Niobium on PM N625" (Senior Design Project - MSE 440 at Purdue University 1993). 